Conference presentation

\title{
Raman spectroscopy reveals distinctive features of drugs at ultra high dilution
}

\section{NC Sukul ${ }^{1,2}$, Tandra Sarkar ${ }^{1,4}$, Atheni Konar ${ }^{1,4}$, Indrani Chakraborty ${ }^{1}$, Achintya Singha $^{3}$, Dipanwita Majumdar ${ }^{3}$, Anirban Sukul ${ }^{1}$.}

${ }^{1}$ Sukul Institute of Homepopathic Research, Santiniketan, West Bengal, India.

${ }^{2}$ Department of Zoology, Visva-Bharati University, Santiniketan W.B., India.

${ }^{3}$ Department of Physics, Bose Institute, Kolkata, W.B. India.

${ }^{4}$ Centre for Healthcare Science and Technology,IIEST, Shibpur, Howrah, W.B., India.

Background: Drugs at ultra high dilution (UHD) have been used in homeopathy for a couple of centuries. The central theme in homeopathic Materia Medica is that each drug has its own distinctive features which need to be matched with the symptoms of a patient for eliciting therapeutic response. However, UHD's very often (above $12 \mathrm{cH}$ ) cross the Avogadro number, and are, therefore, devoid of original drugmolecules. How do they maintain their individual identity? This study aims to address this pertinent que

Objective: The medium of UHD's is ethanol water. It is thought that water structures in a UHD carry the identity of the drug and its rank of dilution. he objective is to decipher the exact nature of water structure in UHes of different drugs by laser Raman spectroscopy.

Method : Six homeopathic drugs and their control ethanol, all in 90\% ethanol v/v, were used in the study. For Sulphur and Natrun m en encied were $30 \mathrm{cH}, 200 \mathrm{cH}$ and $1000 \mathrm{cH}$, and for Calcarea carb and Sepia the potencies $8 \mathrm{cH}, 202 \mathrm{cH}$ and $1002 \mathrm{cH}$. In addition to the four drugs we also used X-ray and Magnetis poli ambo which did not originate from any substance by exposure of ethanol water to $\mathrm{X}$ radiation and strong magnetic field.For this we could use their mother tinctures as well as ultrahigh dilutions(potencies) like $8 \mathrm{cH}, 14 \mathrm{cH}$ and $32 \mathrm{cH}$. The potencies used for ethanol control were $8 \mathrm{cH}, 14 \mathrm{cH}, 32 \mathrm{cH}$ and $20 \mathrm{cH}$. Raman spectra of all the potencies of 6 drugs, ethanol control and pure water were taken in the wave number region of $2400-4200 \mathrm{~cm}^{-1}$. All the samples were reduced to $25 \%$ ethanol by adding appropriate volume of water to each of them before taking the spectra. The mother tinctures MT of X-ray and Magnetis were studied.

The intensity ratio of vibration frequencies between $3200 \mathrm{~cm}^{-1}$ and $3420 \mathrm{~cm}^{-1}$ (R1) and that between $3620 \mathrm{~cm}^{-1}$ and $3420 \mathrm{~cm}^{-1}$ (R2) were calculated for each UHD of the samples.

Results: All the UHD's of the drugs and the control tested show difference in intensities in the stretching vibrations of $\mathrm{CH}$ and $\mathrm{OH}$ groups. The three UHD's from low to high ranks of both Natrum mur and Sulphur show negative relationship with respect to R1 values, and positive one concerning R2 values. R1 values for 3 UHD's of Calcarea carb and Sepia show 
negative and positive relationships, respectively. In case of R2 values the relationship in 3 UHD's is $8<202>1002$ for Calcarea carb, and $8>202<1002$ for Sepia. R1 value position for $\mathrm{X}$-ray is MT $<8,32<14$ and for Magnetis is MT $<8<32<14$. The relative position of R2 values for X-ray is $14<8<32<\mathrm{MT}$ and that for Magnetis is $14<8<32<\mathrm{MT}$. R1 and R2 values for the 2 UHD's $(8 \mathrm{cH}, 202 \mathrm{cH})$ of ethanol control the show negative relationship. For the 3 more UHDs of ethanol control the relative position of $\mathrm{R} 1$ values is $32<8<14$, and for R2 values is $14<8<32$.

Discussion: The intensity of Raman scattering is proportional to the electric dipole-electric dipole polarizability of change. So, the UHD's of different drugs vary from each other with respect to that aspect.

The intensity ratio $\mathrm{R} 1$ gives information about the relative number of $\mathrm{OH}$ groups with strong and weak hydrogen bonds. Judged from the perspective of R1 values, the lower is the rank of UHD, the stronger is the H-bond of the OH groups as with Natrum mur, Sulphur and Calcarea carb. The situation is different with Sepia. Here the higher the potency the stronger is the $\mathrm{H}$-bond of the $\mathrm{OH}$ groups. It is also different with $X$-ray and Magnetis.

The intensity ratio $\mathrm{R} 2$ suggests the relative number of $\mathrm{OH}$ groups with broken and weak hydrogen bonds. In the light of R2, the higher is the UHD rank, the more abundant is the free $\mathrm{OH}$ groups and non-hydrogen bonded water molecules, as with Natrum mur, Sulphur . However, for Calcarea carb and Sepia the proportion of free water molecules is not positively correlated with the potency ranks studied. The same is the situation with Xray and Magnetis. As for the control the UHD's differ from each other with respect to free water molecules and hydrogen bond strength. It may be mentione here that UHD of ethanol is a homeopathic drug with specific symptoms.

Conclusion: UHD's of homeopathic drugs irrespective of their origin either from any substance or energy, are different from da h d ther thit of $\mathrm{OH}$ groups and relative proportiфn fre water hecules. These two physical aspects characterize the uniqueness of homeopathic drugs vis-à-vis the central theme in homeopathic material medica.

(C) International Journal of High Dilution Research. Not for commercial purposes. 